\title{
Sacrificial Layer Technique for Releasing Metallized Multilayer SU-8 Devices
}

\author{
Anand Tatikonda ${ }^{1}$, Ville P. Jokinen ${ }^{1}$, Hanno Evard ${ }^{2}$ and Sami Franssila ${ }^{1, *}$ \\ 1 Department of Chemistry and Materials Science, School of Chemical Engineering, Aalto University, \\ 02150 Espoo, Finland; anand.tatikonda@aalto.fi (A.T.); ville.p.jokinen@aalto.fi (V.P.J.) \\ 2 Division of Pharmaceutical Chemistry and Technology, Faculty of Pharmacy, University of Helsinki, \\ 00014 Helsinki, Finland; evardhanno@gmail.com \\ * Correspondence: sami.franssila@aalto.fi
}

Received: 15 November 2018; Accepted: 10 December 2018; Published: 19 December 2018

\begin{abstract}
The low fabrication cost of SU-8-based devices has opened the fields of point-of-care devices (POC), $\mu$ TAS and Lab-on-Chip technologies, which call for cheap and disposable devices. Often this translates to free-standing, suspended devices and a reusable carrier wafer. This necessitates a sacrificial layer to release the devices from the substrates. Both inorganic (metals and oxides) and organic materials (polymers) have been used as sacrificial materials, but they fall short for fabrication and releasing multilayer SU-8 devices. We propose photoresist AZ 15nXT (MicroChemicals GmbH, Ulm, Germany) to be used as a sacrificial layer. AZ 15nXT is stable during SU-8 processing, making it suitable for fabricating free-standing multilayer devices. We show two methods for cross-linking AZ $15 \mathrm{nXT}$ for stable sacrificial layers and three routes for sacrificial release of the multilayer SU-8 devices. We demonstrate the capability of our release processes by fabrication of a three-layer free-standing microfluidic electrospray ionization (ESI) chip and a free-standing multilayer device with electrodes in a microchannel.
\end{abstract}

Keywords: SU-8; multi layered chips; AZ 15nXT; sacrificial release; microchips; microfabrication; microfluidics; free standing

\section{Introduction}

SU-8 has gained wide acceptance in micro- and nanofabrication [1] due to its superior mechanical [2] and thermal stability [3] and excellent bio-chemical compatibility [4]. SU-8 excels over most other polymers due to its mechanical properties, e.g., modulus of elasticity of $5 \mathrm{GPa}$ [2] and the ability to easily fabricate high aspect ratio structures (up to 50:1) [5-8]. It is also easy to fabricate multilayer structures, with multiple spin-coating and exposure steps, and a single development step. SU-8 can be bonded to itself, resulting in channels with four identical walls [9]. There are a wealth of applications of SU-8 in $\mu$ TAS/Lab-on-chip technologies [10,11].

In many cases, free-standing devices are needed. For instance, when sharp tips must be made, as in nozzle and spraying devices, the substrate must be removed. A silicon substrate is also a drawback in applications where high electric fields are used, like capillary electrophoresis. SU-8 is translucent, and sometimes this is used. Two main routes exist for release: sacrificial wafer process, and sacrificial layer processes [12]. Carrier wafer sacrifice is costly and slow, and we do not consider it in this work.

The basic requirements for a sacrificial layer include easy deposition and patterning, chemical, thermal and mechanical stability during subsequent processing steps, and selective etching in the release step [13]. Many materials have been employed as sacrificial layers: metals such as copper, chromium and aluminum [14-17], silicon dioxide [9,18,19] and polymers [20-23]. Polymers include polystyrene, PDMS (Polydimethylsiloxane), SAMs (self-assembled molecules), OmniCoat, PMGI 
(Polydimethylglutarimide) and various resists such as AZ and un-cross-linked SU-8 itself [15,21,24-27]. The drawback of inorganic sacrificial layers is the added complexity of thin film deposition and etching. They are often limited in thickness to ca. $1 \mu \mathrm{m}$ due to slowness of chemical vapor deposition (CVD) and physical vapor deposition (PVD) deposition methods. They are, however, stable and allow different release etch processes to be implemented, e.g., $\mathrm{HCl}$ for copper or $\mathrm{NaOH}$ for aluminum. Unfortunately they fall short for full wafer release as they generate internal stresses and might lead to micro cracks [24]. Even more important, if the device contains metallization, the release etch might remove the metallization simultaneously. Almost all applications that require metallization of the SU-8 devices use polymer-based sacrificial layers [13,21,28,29].

Polymer-based sacrificial layers can be easily integrated into the microfabrication processes. Spin-coating and development steps are quick and sometimes they can be combined beneficially with the SU-8 processing. Thickness of a spin coated layer can be varied over a larger range than that of CVD or PVD layers. The biggest drawback of polymeric sacrificial layers for metallized multilayer devices is their poor thermal and chemical stability during SU-8 processing [15]. Most polymers are not stable against thermal stresses encountered during metal deposition, resulting in degradation and early releasing of the structure. In [21] it was shown that extreme care has to be taken for metallization integrity on SU-8 structures when using polymer sacrificial layers. OmniCoat, which is a commercially available special polymer developed as a sacrificial layer for SU-8 also falls short, as its not stable when fabricating full wafer multilayer SU-8 microfluidic devices [23,28,30]. Few groups have used common photoresists as sacrificial layers, but they all fall short as these resists are not stable in SU-8 developers for multilayer microfluidic devices, or when very long process times are needed [30].

Addressing these issues, we show that the negative photoresist AZ 15nXT (MicroChemicals $\mathrm{GmbH}, \mathrm{Ulm}$, Germany) as the release layer allows the fabrication of free-standing multilayer devices with metallization. A wafer scale process is presented, exploiting the inertness and thermal stability of AZ 15nXT in SU-8 processing. The release etch is done TechniStrip NI555 solvent (MicroChemicals $\mathrm{GmbH}, \mathrm{Ulm}$, Germany). We show two example processes: fabrication of a three-layer free-standing microfluidic electrospray ionization (ESI) chip and a free-standing multilayer device with electrodes in a microchannel.

\section{Materials and Methods}

\subsection{Chemical and Materials}

Single-side polished $100 \mathrm{~mm}$ diameter $<100>$ orientation silicon wafers were purchased from Siegert wafer $\mathrm{GmbH}$ (Aachen, Germany). SU-8 negative photoresist (SU-8 50) and developer (mr-DEV-600) were purchased from Microresist Technologies GmbH (Berlin, Germany). AZ 5412E, AZ 15nXT (450 CPS), MIF 312 developer and TechniStrip ${ }^{\circledR}$ NI555 was purchased from Microchemicals $\mathrm{GmbH}$ (Ulm, Germany). OmniCoat ${ }^{\mathrm{TM}}$ and PMGI are purchased from MicroChem Corp. (Westborough, MA, USA). Buffered HF and isopropanol were purchased from Honeywell International Inc., (Kuopio, Finland). Methanol, formic acid, and verapamil for mass spectrometry were purchased from Sigma Aldrich (Espoo, Finland). Polymer sheets used as carriers for bonding purposes were purchased from Arron (Helsinki, Finland). Chrome glass masks for ESI chips were acquired from ML\&S GmbH (Greifswald, Germany) and film masks for metalized microchannels were from Micro Lithography Services Limited (Chelmsford, UK). All the reagents for clean room purposes are VLSI grade and for mass spectroscopy they are LC-mass spectrometry (MS) grade.

\subsection{Microfabrication}

Silicon wafers were used as substrates for microfabricating SU-8 multilayers. Resist AZ 15nXT was used as a sacrificial layer underneath the first SU-8 layer. The ESI device contains three layers of SU-8 defined by three lithography steps. The ESI device design and the microfabrication of the first two layers of SU-8 and adhesive bonding of the third layer have been reported previously [31]. All the 
microfabrication is performed inside a cleanroom and only sacrificial release was done in a chemical hood outside cleanroom.

Multilayer SU-8 fabrication process on silicon is quite challenging due to the large mismatch in thermal expansion coefficients. This generates stresses at the interface, easily resulting in adhesion loss. The problem is compounded when more SU-8 layers are added. To ensure resist adhesion, native oxide was removed from silicon surface in buffered HF solution. Wafers were rinsed in DI water and dried by nitrogen blow gun, followed by baking at $200{ }^{\circ} \mathrm{C}$ for a minimum of $15 \mathrm{~min}$ to dehydrate the silicon surface.

SU-8 and AZ 15nXT dispensing was done by manual pipetting. Baking was performed on a programmable hot plate HP-220 from Unitemp GmbH (Germany). Sacrificial layer of AZ 15nXT of $4 \mu \mathrm{m}$ thickness was spin coated (5000 rpm) on a cleaned silicon wafer and baked at $160{ }^{\circ} \mathrm{C}$ for $15 \mathrm{~min}$ or soft baked for $110{ }^{\circ} \mathrm{C}$ for $3 \mathrm{~min}$ and flood UV exposed under mask aligner. The first SU-8 layer (70 $\mu \mathrm{m}$ thick) was applied on top of the cured AZ 15nXT. In our inverted fabrication process this first SU-8 layer will form the roof of the channel in the final device.

For the $70 \mu \mathrm{m}$ thick (2000 rpm) SU-8 layer the ramping rate of $20^{\circ} \mathrm{C} / \mathrm{min}$ was used to bring the wafer from room temperature to $65^{\circ} \mathrm{C}$, after which there was a holding step of $15 \mathrm{~min} .10^{\circ} \mathrm{C} / \mathrm{min}$ ramping was then applied to raise the temperature to $95^{\circ} \mathrm{C}$. The hold time at $95{ }^{\circ} \mathrm{C}$ was again $15 \mathrm{~min}$. After cooling down to room temperature, the prebaked SU-8 layer was UV exposed $(\lambda=365 \mathrm{~nm})$ through a photomask with $660 \mathrm{~mJ} / \mathrm{cm}^{2}$ (22 s exposure) in a Suss MicroTech MA-6 mask aligner. Post exposure baking of the first SU-8 layer is done by ramping from room temperature to $95^{\circ} \mathrm{C}$ at $20^{\circ} \mathrm{C} / \mathrm{min}$ and baking it for $12 \mathrm{~min}$ and then cooling down to room temperature at rate of $3.75^{\circ} \mathrm{C} / \mathrm{min}$. After cooling down to room temperature, the SU-8 layer was developed in mr-Dev-600 developer for $10 \mathrm{~min}$, washed in isopropanol, and dried. Schematic patterns of different layers are show in Figure 1.

(a)
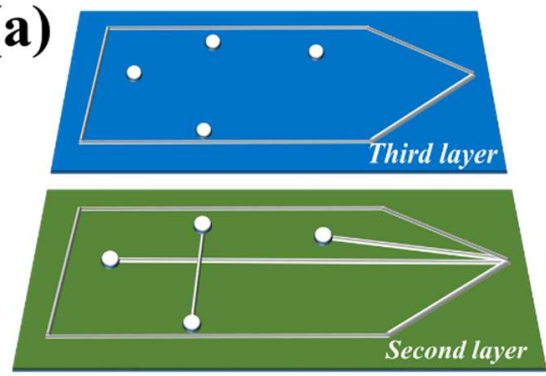

Multilayered SU-8 chip (b)
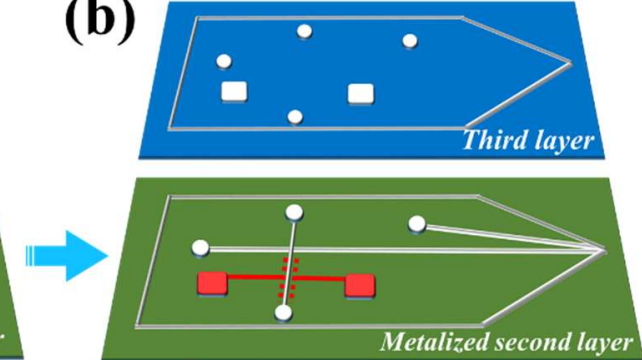

SU-8 chip with metalized channels

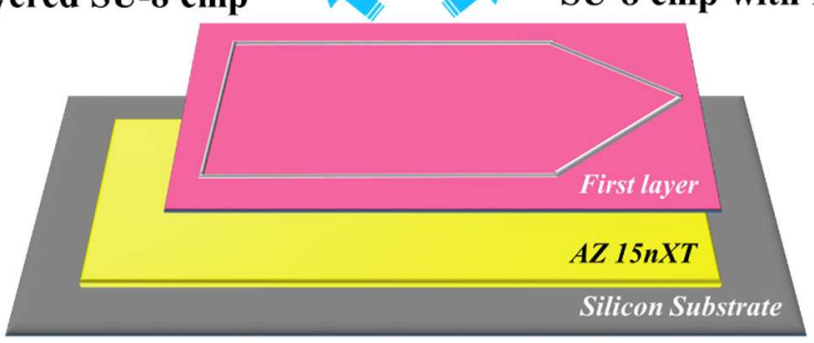

Figure 1. Schematics of multilayer SU-8 free-standing microfluidic device for mass spectroscopy electro spray ionization. (a) the multilayered SU-8 ESI chip (b) SU-8 chip with extra metallization step.

The second SU-8 layer defines the $50 \mu \mathrm{m}$ wide microchannel. It is aligned to the sharp tip in the first SU-8 layer. The thickness of the second layer SU-8 layer is $50 \mu \mathrm{m}$ (3000 rpm) which defines the depth of the microchannel (Figure 1). The prebake ramp rates for the second layer were similar to the first layer, but the hold times were only $5 \mathrm{~min}$ at $65^{\circ} \mathrm{C}$ and $3 \mathrm{~min}$ at $95^{\circ} \mathrm{C}$. Exposure dose was $450 \mathrm{~mJ} / \mathrm{cm}^{2}$ (15 s exposure). Post exposure bake of the second layer is identical to the first layer. After cooling down the second layer was developed for $10 \mathrm{~min}$, rinsed, and dried as before. 
The metallization of the microchannel was performed after the second SU-8 layer processing (Figure 1b). Lift-off patterning used AZ 5412E with standard parameters. Sputtering of $5 \mathrm{~nm}$ of $\mathrm{Cr}$ and 200 nm Al was done in Plasmalab 400 (Oxford Instruments, Abingdon, UK). The sputtered wafer was immersed in acetone for lift-off (Figure 2b).

(a)

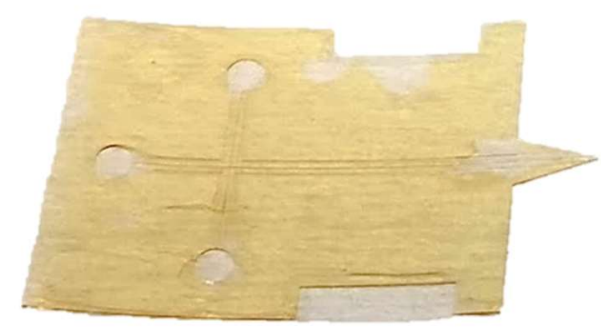

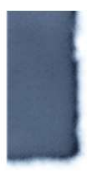

(b)

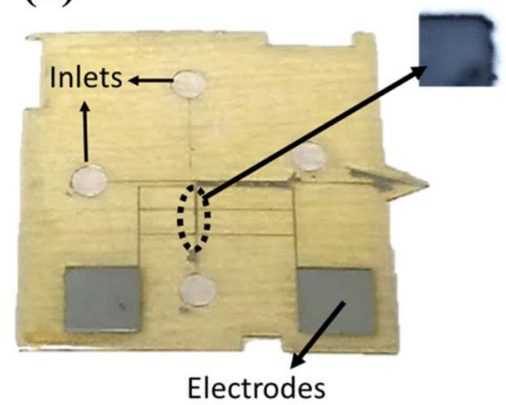

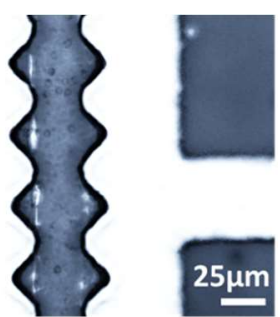

Metalized Channel

Figure 2. Microfabricated multilayer SU-8 chips (a) the multilayered SU-8 electrospray ionization (ESI) chip for mass spectrometer analysis (b) metalized SU-8 chip with electrodes. The insert shows the channels which are metalized by lift-off.

The third layer forms the inlets of the chip. A flexible polymer sheet was cleaned with acetone and isopropanol to remove any residual particles to ensure defect-free SU-8 layer. This polymer sheet was then attached to a silicon carrier wafer with double-sided tape. The third layer of SU-8, $70 \mu \mathrm{m}$ thick, was spin coated. Prebaking conditions were identical to first layer of SU-8 except the cooling step, which was at a rate of $3.2^{\circ} \mathrm{C} / \mathrm{min}$ from $95^{\circ} \mathrm{C}$ to $63^{\circ} \mathrm{C}$ and then held at this temperature for $20 \mathrm{~min}$. Simultaneously the silicon wafer with $15 \mathrm{nXT}$ and the first and the second SU-8 layer was heated to $63{ }^{\circ} \mathrm{C}$. The polymer sheet was then released from the tape on the carrier wafer and bonding SU-8 layer is placed carefully on top of the second layer of SU-8 and laminated with minimal force to improve adhesion between the layers. This leaves the non-exposed 3rd SU-8 layer under polymer sheet in contact with the exposed 2nd layer. The wafer stack was kept on hot plate at $63{ }^{\circ} \mathrm{C}$ for $15 \mathrm{~min}$ and then freely cooled to room temperature. The third layer was exposed with dose of $750 \mathrm{~mJ} / \mathrm{cm}^{2}(25 \mathrm{~s}$ exposure) through the polymer sheet. After post bake the polymer sheet was peeled off. Development of the 3rd layer was identical to the 1st layer. The ESI chips (Figure $2 \mathrm{a}$ ) are of $2.2 \mathrm{~cm} \times 3 \mathrm{~cm}$ and the metalized chips (Figure $2 \mathrm{~b}$ ) are $2 \mathrm{~cm} \times 2.5 \mathrm{~cm}$ with both having channels of $50 \mu \mathrm{m}$ deep and width.

\subsection{Cross-Linking and Release Etching}

Sacrificial layer AZ 15nXT was used for releasing multilayer SU-8 microfluidic devices from the silicon wafer. For all the chip designs we used $4 \mu \mathrm{m}$ thick AZ 15nXT. We have used two different methods for cross-linking of the AZ 15nXT. AZ 15nXT, being a negative photoresist, is cross-linked by UV exposure or by heating at elevated temperatures. The UV exposure was performed by flood exposure of $2800 \mathrm{~mJ} / \mathrm{cm}^{2}$ in the same MA-6 mask aligner as the SU-8 exposures, and the thermal curing was done at $160{ }^{\circ} \mathrm{C}$ on a hot plate for $15 \mathrm{~min}$.

For releasing, we demonstrate three different sacrificial etch techniques. In first method the SU-8 processed wafer was immersed in room temperature TechniStrip NI555 for overnight. In the second method, TechniStrip was heated to $60^{\circ} \mathrm{C}$. The third method involved a thermal shock process where the processed wafer was rapidly heated to $120{ }^{\circ} \mathrm{C}$ on a hot plate for $20 \mathrm{~s}$ and placed immediately in TechniStrip solution which was held at RT. The holding time of the wafer in Technistrip was determined by waiting until the chips start to float to the top of solution. We also compared the 
sacrificial release of SU-8 layers with commercially available polymer materials OmniCoat and PMGI. For these comparison tests we have spin coated, baked and exposed OmniCoat and PMGI according to parameters provided by MicroChem Corp. [32,33].

\subsection{Mass Spectrometry Measurements}

Agilent 6300 Series Ion Trap (Santa Clara, CA, USA) was used for these experiments. Capillary voltage of $-1600 \mathrm{~V}$ was applied and mass range of 100 to $600 \mathrm{~m} / \mathrm{z}$ was scanned. Drying gas temperature was set to $25^{\circ} \mathrm{C}$ and flow set to $3 \mathrm{~L} / \mathrm{min}$. The SU-8 electrospray chip was placed onto the XYZ stage and positioned so that the distance between electrospray tip and the cone was 2-3 mm. A platinum electrode connected to a high voltage source (built in-house) was placed into the solvent well of the chip. $1500 \mathrm{~V}$ (compared to ground) was applied to the electrode after which $2 \mu \mathrm{L}$ of spray solvent (90\% MeOH, 10\% Milli-Q water (Merck Millipore, Burlington, MA, USA), which both contained $0.5 \%$ formic acid) was added to the well. In the case of verapamil measurements, $400 \mathrm{nM}$ solution of the analyte in spray solvent was prepared from verapamil hydrochloride.

\section{Results and Discussion}

To have a free-standing embedded microfluidic channel made entirely of SU-8, we need three layers of SU-8, patterned on top of each other, aligned and bonded together [19]. The sacrificial layer is the most critical and challenging step, as it determines the release etch. The addition of metallization adds its own peculiarities as it limits the kinds of sacrificial layers and etchants that can be used.

The rationale behind using the AZ $15 \mathrm{nXT}$ as sacrificial layer was that it is a widely used resist for electroplating. For this purpose, AZ 15nXT is inert for acidic condition and cyclic electrical fields which are encountered during electroplating process. Therefore, AZ 15nXT crosslinks into highly stable state, which also makes it resistant against most common developers and solvents used in microfabrication. Stripping AZ 15nXT is accomplished by a specifically selected solvent TechniStrip ${ }^{\circledR}$ NI555 which is a mixture of acidic-based solvents [34]. The stability of AZ 15nXT depends on the extent of cross-linking. Once the AZ 15nXT has been cross-linked by either the UV radiation or thermal means, it is highly stable over wide range of conditions. Specifically, it does not dissolve or swell in solvents of SU-8 process or mr-DEV-600 a SU-8 developer. This is different from other common resists which have been used as sacrificial layers before [15,25]. For ESI devices the total development time for three SU-8 layers was $30 \mathrm{~min}$ and for metalized chip we have total 33 min development times. The recommended soft bake for $15 \mathrm{nXT}$ is $3 \mathrm{~min}$ at $130{ }^{\circ} \mathrm{C}$. However, during multilayer SU-8 processing, thermal cross-linking was done at $160{ }^{\circ} \mathrm{C}$ for at least $15 \mathrm{~min}$. Due to high degree of cross-linking by $\mathrm{UV}, \mathrm{nXT}$ is stable during the metal deposition process. UV-assisted cross-linking of AZ 15nXT slows the dissolution in TechniStrip, for this reason UV cross-linking of AZ 15nXT was used only when metallization of SU-8 devices was needed (Figure 1b). Thermal cross-linking of AZ 15nXT was done by baking the resist above its post bake temperature. We have tried temperatures and times ranging from $120^{\circ} \mathrm{C}$ to $200{ }^{\circ} \mathrm{C}$ for times $3 \mathrm{~min}$ to $30 \mathrm{~min}$ and found $160^{\circ} \mathrm{C}$ and $15 \mathrm{~min}$ baking to be optimal for our process. This is based on stability of the AZ 15nXT in multiple SU-8 developing steps and the ease of releasing in Technistrip NI555. AZ 15nXT exhibited excellent adhesion to silicon and SU-8 layers as well as high stability in SU-8 developer after cross-linking (Figures 3a and 4a,b).

In the first release method (Method 1), the whole silicon wafer with the SU-8 multilayer structure (with or without metallization) was immersed in TechniStrip NI555 overnight. AZ 15nXT slowly swells and dissolves in TechniStrip. TechniStrip also penetrates the underlying $\mathrm{nXT}$ releasing individual chips. However, the rate of dissolution of the AZ 15nXT in TechniStrip is slow making the release process time consuming. 


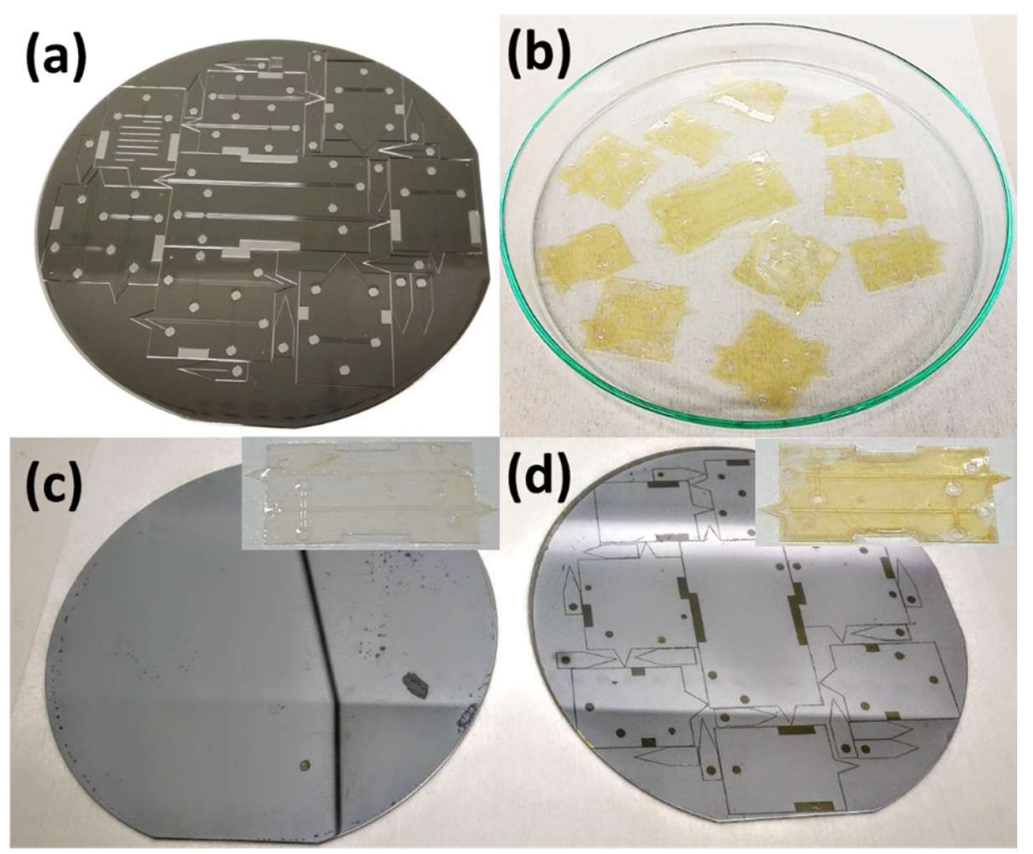

Figure 3. (a) Multilayer SU-8 devices fabricated on AZ $15 \mathrm{nXT}$ on silicon wafer before release (b) Free-standing SU-8 ESI chips after releasing from the substrate (c) Silicon wafer after releasing by wet Technistrip assisted release (Method 1 or 2), which leaves very little residue and can be reused with simple cleaning steps (d) silicon wafer after releasing from thermal assisted Technistrip release (Method 3), which leaves more residues. Insert shows the released chips. Residual AZ 15nXT from chips can be removed by incubating for more time in Technistrip.

In the second method (Method 2), we used heated TechniStrip at $60^{\circ} \mathrm{C}$. This releases SU-8 chips in less than $3 \mathrm{~h}$. The silicon carrier wafer released by both methods one and two show very little residues of AZ 15nXT, making them reusable with minimal cleaning (Figure 3b,c).

To release the chips much faster we have developed a third method (Method 3), which is based on thermal shock release of AZ 15nXT. In this technique, the silicon wafer with SU-8 layers was heated on a hot plate for $20 \mathrm{~s}$ at $120^{\circ} \mathrm{C}$ and placed immediately into TechniStrip solvent which was held at room temperature. Within $15 \mathrm{~min}$ individual SU-8 chips were released from the silicon substrate (Figure 3b,d). Due to thermal expansion difference between silicon $\left(2.6 \mathrm{ppm} /{ }^{\circ} \mathrm{C}\right)$ and the SU-8 $\left(52 \mathrm{ppm} /{ }^{\circ} \mathrm{C}\right)$, thermal stresses developed at the $15 \mathrm{nXT}$ layer between silicon and the first SU-8 layer. Thermally assisted release process leaves more contamination on the silicon wafer, which can be removed by incubating the released silicon wafers in TechniStrip followed by Piranha cleaning.

According to the MicroChem corp. it takes $20 \mathrm{~min}$ for AZ 15nXT to swell and completely dissolve in the TechniStrip solution. These are for cross-linked AZ 15nXT from the manufacturer conditions and it takes much longer for relatively heavily cross-linked AZ 15nXT and would need heating of the TechniStrip or even sonication of the solution during release, which might damage microfabricated devices [34]. However, the processing conditions required for multiple layers of SU-8 modify the resist, making it much harder to remove. The exact swelling/dissolving rate is not known, but it was at least stable for more than $30 \mathrm{~min}$ in SU-8 developers.

OmniCoat $^{\mathrm{TM}}$ is recommended by the MicroChem corp. as a sacrificial layer for SU-8 microfabrication [33]. It is ideal as a sacrificial layer for microfabricating single layer of SU-8 or multilayers without any sealed or embedded structures but OmniCoat falls short when used for multilayer processes [30]. It was reported earlier that OmniCoat has a poor adhesion with the silicon and SU-8 making resulting in higher stresses and delaminating the SU-8 structures [35]. This is ideal for single layer SU-8 whereas for multilayers it falls short, as the stresses would delaminate during the processing. PMGI is a lift of resist which is commonly used for sacrificial release in MEMS [36-38]. 
Thermally cured PMGI is not suitable for multilayer sacrificial release and needs deep-UV exposure to make it inert for SU-8 developer [38].

\subsection{Comparison between AZ $15 n X T$, OmniCoat and PMGI}

We have compared our AZ 15nXT release process with OmniCoat ${ }^{\mathrm{TM}}$ and PMGI, which are commercially available polymer sacrificial layers for SU-8 process. The first layer of SU-8 was spin coated, soft baked, exposed, and post baked identically on all three-release layer materials. OmniCoat and PMGI wafers were placed in SU-8 developer and within minutes, individual chips were released from the silicon wafer (Figure $4 \mathrm{c}, \mathrm{d}$ ). This suggests that OmniCoat and PMGI are not stable in SU-8 developer and are only suitable for single layer of SU-8 process.
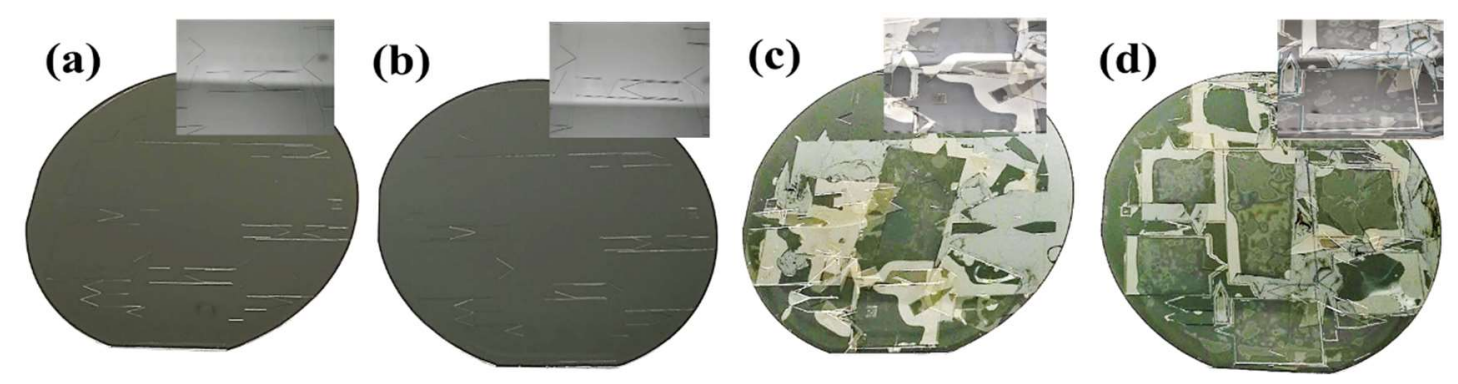

Figure 4. Multilayer SU-8 devices fabrication, during the first layer development (a) AZ 15nXT thermally cross-linked (b) AZ 15nXT UV cross-linked (c) Omni coat and (d) PMGI in place of AZ 15nXT. The SU-8 surfaces have delaminated from the silicon substrate when we have OmniCoat or PMGI are used as sacrificial layers making the multilayer process impossible. The first layer is quite stable when we have used AZ 15nXT as sacrificial layer. Insert shows the zoomed in section of the wafer where in $(\mathbf{a}, \mathbf{b})$ the pattern is intact in $(\mathbf{c}, \mathbf{d})$ the patterns have peeled off from the surface.

We tried to optimize release layer thickness, exposure and baking temperatures but could not improve OmniCoat or PMGI for multilayer process. The sacrificial layer encounters multiple solvent and thermal steps during microfabrication. Since single release etch step remains the most viable option for practically all devices, the sacrificial material must tolerate multilayer processes. AZ 15nXT is robust and superior to any other polymer-based sacrificial release layer for multilayer SU-8 structures. The stability of $15 \mathrm{nXT}$ can be tailored easily to suit any process by simply changing the thickness, baking time or UV exposure making it adoptable to other multilayer applications. Sacrificial etching of long channels from the mouth of the channel is not a viable option because of excessive etch times (overnight or several days) and SU-8 deformation [18].

Schmidt et al. [28] have shown that OmniCoat can be used for SU-8 multilayer process in which sacrificial release was performed using common clean room developer MIF-319. This limits the selection of photoresists that can be used for microfabrication and making the metallization process much more challenging. Valencia et al. [37], and Bohl [30] have shown that PMGI and OmniCoat respectively can be used for processing SU-8 multilayers where all the SU-8 layers are developed together in one single step. This is not suitable for embedded microchannel as it would take long development time for SU-8 in embedded micro channels [18]. Foulds et al. [38] have shown that PMGI can be used as sacrificial layer for making free-standing multilayer SU-8 MEMS. The biggest problem arising was the cracking of PGMI itself resulting in defects in the SU-8 layers and other lithography process which are microfabricated on PGMI surface.

Polymer-based sacrificial layers which have been developed for MEMS applications where the areas to be released are fairly small, usually device element size, but in microfluidics release is often about full chips or even wafers. Because of this difference, sacrificial materials which are good for MEMS, such as PGMI [38], cannot be used for microfluidic devices (Figure 4d). This effect is more pronounced when we have multilayer SU-8 process. 


\subsection{ESI Chip}

To test the stability of thermal cross-linked AZ 15nXT sacrificial layer we have microfabricated multilayer SU-8 microfluidic device for MS. Free-standing SU-8 chips are used for detecting biomarkers by MS. The chip contains application reservoir, a microfluidic channel, and a sharp tip for spraying. A sharp tip is needed to establish a stable Taylor-cone. The droplets are ionized either by a corona needle or an UV-lamp. The functionality and performance of these free-standing SU-8 ionization chips were evaluated by analyzing molecules of verapamil by mass spectrometry. The limit of detection (LOD) was measured with injection under Selective Reaction Monitoring (SRM) using two pre-selected precursor/product ion pairs of $m / z 455 \rightarrow 165$ and $455 \rightarrow 303$. The LOD we have achieved was $3.62 \mathrm{nM}$ with a good correlation factor of 0.97 (Figure $5 b$ ). These are similar or even better to what we have reported with similar SU-8 multilayer devices $[25,26,36,38]$. We speculate that this is due to reduced etching times and complete dissolution of the sacrificial layer, i.e., fewer residues. SU-8 devices released with the AZ 15nXT as sacrificial layer behave consistently compared to the devices which are released by other methods $[18,19,31,39]$.
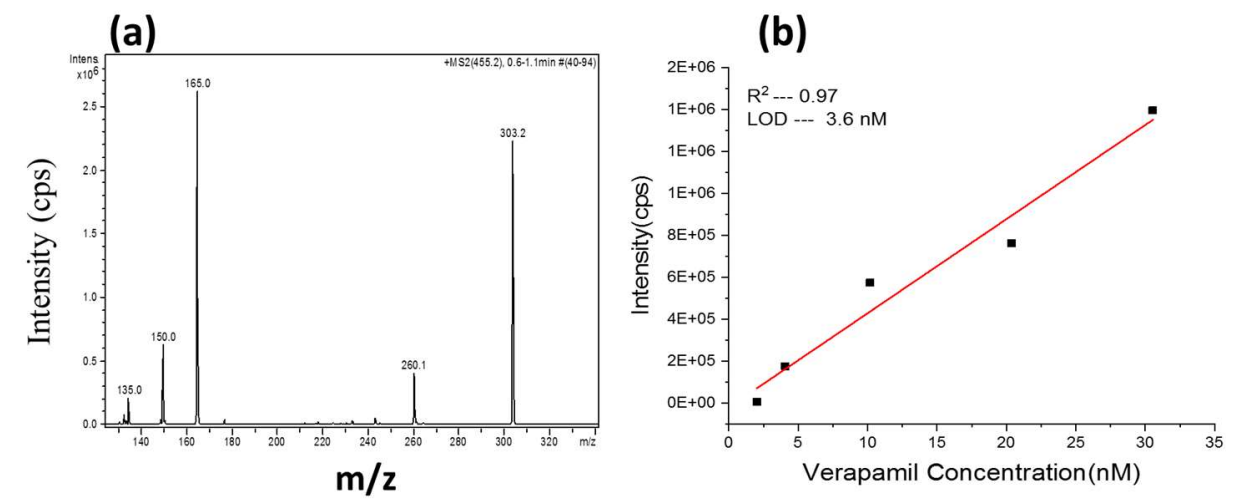

Figure 5. MS spectra for Verapamil (a) internal standards were protonated verapamil at $\mathrm{m} / \mathrm{z} 455.3$

(b) Limit of detection for $\mathrm{S} / \mathrm{N}$ of 2.5 was $3.6 \mathrm{nM}$ with correlation coefficient of 0.97 .

\subsection{Free-Standing Metallized Chip}

To test the stability of AZ 15nXT as sacrificial layer in metallization process, we have used UV cross-linked AZ 15nXT. After the patterning of the first and second layer of SU-8, we have metallized the microchannel with $\mathrm{Cr} / \mathrm{Al}(5 \mathrm{~nm} / 200 \mathrm{~nm}$ ) (Figure 1b). After lift-off patterning of the metal electrodes on the second SU-8 layer, we bonded the lid of the microchannel as before, and released the chips by the first method (Method 1) in TechniStrip (Figure 2b). For the metallization process, thermally cross-linked AZ 15nxt was not stable in sputtering process. The UV cross-linked AZ 15nXT could withstand the thermal stresses caused by the sputtered layers and handle the process solvents in the metal patterning step. Inorganic sacrificial layers such as metals and oxides are not compatible for microfabrication of metallized structures. Acidic process such as $\mathrm{HF}[9,19]$ are needed for oxide releasing or $\mathrm{HCl}$ for metal release, and these adversely affect the integrity of the metalized SU-8 structures. This limits the choice of sacrificial layers to organics for metalized SU-8 chips. This section may be divided by subheadings. It should provide a concise and precise description of the experimental results, their interpretation as well as the experimental con clusions that can be drawn.

Based on the optimization, we propose the following two optimized processes to be use for residual-free release and fast release of SU-8. For residual-free release the best method was to spincoat $4 \mu \mathrm{m}$ of AZ $15 \mathrm{xNT}$ and bake at $160^{\circ} \mathrm{C}$ for $15 \mathrm{~min}$ followed by process the multilayer SU-8 devices on AZ 15nXT and place the processed silicon wafer in TechniStrip solution at $60^{\circ} \mathrm{C}$ during the release (Method 2). Thermal shock (Method 3) is best for fast release of the SU-8 chips process as before. The extent of AZ 15nXT stability depends on the thickness of the film, UV exposure dose and time, and the bake temperature. We have optimized all these parameters to achieve highly stable AZ 15nXT 
sacrificial layer for our multilayer SU-8 process at the same time keeping in mind the ease of full wafer release of the devices in TechniStrip.

\section{Conclusions}

We have developed a novel fabrication process where negative resist AZ 15nXT serves as a sacrificial layer for multilayer SU-8 process. Using AZ 15nXT resist will significantly reduce the processing steps needed to make released devices. For residual-free release of multilayer SU-8 devices the best method is to place the processed silicon wafer with SU-8 structures in heated TechniStrip solution and for fast release thermal shock method is the best solution. We have demonstrated the performance of the released SU-8 devices in mass spectrometric analysis of verapamil with reproducible spraying and low limit of detection.

AZ 15nXT is a good compromise between stability during device processing and reasonably fast release. While OmniCoat and PGMI can be used to release simple SU-8 chips, our process is suitable for any geometry and any number of SU-8 layers. Our general release method will help in stable integration of metallization steps into the polymer MEMS and can readily be used for other microfabrication process.

Author Contributions: Conceptualization, A.T.; methodology, A.T.; validation, A.T. and H.E.; formal analysis, A.T. and H.E.; investigation, A.T. and V.P.J.; writing-original draft preparation, A.T. and V.P.J.; writing-review and editing, A.T., V.P.J. and S.F.; supervision, V.P.J. and S.F.; project administration, S.F.; funding acquisition, S.F.

Funding: This research was funded by 3iRegeneration project. Financial support was provided by TEKES Large Strategic Research Opening project (40313/95, A.T., H.E.).

Acknowledgments: We would like to thank Risto Kostiainen and Tapio Kotiaho from University of Helsinki for helping us with the mass spectrometry facility. The work used the cleanroom facilities of Micronova, Aalto University.

Conflicts of Interest: The authors declare no conflict of interest.

\section{References}

1. Lorenzdag, H.; Despont, M.; Fahrnidag, N.; LaBianca, N.; Renauddag, P.; Vettiger, P. SU-8: A low-cost negative resist for MEMS. J. Micromech. Microeng. 1997, 7, 121-124. [CrossRef]

2. Dellmann, L.; Roth, S.; Beuret, C.; Racine, G.A.; Lorenz, H.; Despont, M.; Renaud, P.; Vettiger, P.; De Rooij, N.F. Fabrication process of high aspect ratio elastic and SU-8 structures for piezoelectric motor applications. Sens. Actuators A Phys. 1998, 70, 42-47. [CrossRef]

3. LaBianca, N.C.; Gelorme, J.D. High-aspect-ratio resist for thick-film applications. Proc. SPIE 1995, 2438, 846-852. [CrossRef]

4. Stangegaard, M.; Wang, Z.; Kutter, J.P.; Dufva, M.; Wolff, A. Whole genome expression profiling using DNA microarray for determining biocompatibility of polymeric surfaces. Mol. Biosyst. 2006, 2, 421-428. [CrossRef]

5. Vora, K.D.; Peele, A.G.; Shew, B.Y.; Harvey, E.C.; Hayes, J.P. Fabrication of support structures to prevent SU-8 stiction in high aspect ratio structures. Microsyst. Technol. 2007, 13, 487-493. [CrossRef]

6. Dentinger, P.M.; Krafcik, K.L.; Simison, K.L.; Janek, R.P.; Hachman, J. High aspect ratio patterning with a proximity ultraviolet source. Microelectron. Eng. 2002, 61-62, 1001-1007. [CrossRef]

7. Williams, J.D.; Wang, W. Using megasonic development of SU-8 to yield ultra-high aspect ratio microstructures with UV lithography. Microsyst. Technol. 2004, 10, 694-698. [CrossRef]

8. Zagrodzinski, J. Solitons and wavetrains: Unified approach. J. Phys. A Math. Gen. 1984, 17, 3315-3320. [CrossRef]

9. Tuomikoski, S.; Franssila, S. Free-standing SU-8 microfluidic chips by adhesive bonding and release etching. Sens. Actuators A Phys. 2005, 120, 408-415. [CrossRef]

10. Marie, R.; Schmid, S.; Johansson, A.; Ejsing, L.; Nordström, M.; Häfliger, D.; Christensen, C.B.V.; Boisen, A.; Dufva, M. Immobilisation of DNA to polymerised SU-8 photoresist. Biosens. Bioelectron. 2006, 21, 1327-1332. [CrossRef]

11. Pai, J.H.; Wang, Y.; Salazar, G.T.A.; Sims, C.E.; Bachman, M.; Li, G.P.; Allbritton, N.L. Photoresist with low fluorescence for bioanalytical applications. Anal. Chem. 2007, 79, 8774-8780. [CrossRef] [PubMed]

12. Franssila, S. Introduction to Microfabrication, 2nd ed.; Wiley: West Sussex, UK, 2010; ISBN 9781119990413. 
13. Cui, Z.; Lawes, R.A. A new sacrificial layer process for the fabrication of micromechanical systems. J. Micromech. Microeng. 1997, 7, 128-130. [CrossRef]

14. Seidemann, V.; Rabe, J.; Feldmann, M.; Buttgenbach, S. SU8-micromechanical structures with in situ fabricated movable parts. Microsyst. Technol. 2002, 8, 348-350. [CrossRef]

15. Psoma, S.D.; Jenkins, D.W.K. Comparative assessment of different sacrificial materials for releasing SU-8 structures. Rev. Adv. Mater. Sci. 2005, 10, 149-155.

16. Nguyen, N.T.; Truong, T.Q. A fully polymeric micropump with piezoelectric actuator. Sens. Actuators B Chem. 2004, 97, 137-143. [CrossRef]

17. Nguyen, N.T.; Truong, T.Q.; Wong, K.K.; Ho, S.S.; Lee-Ngo Low, C. Micro check valves for integration into polymeric microfluidic devices. J. Micromech. Microeng. 2004, 14, 69-75. [CrossRef]

18. Tuomikoski, S.; Sikanen, T.; Ketola, R.A.; Kostiainen, R.; Kotiaho, T.; Franssila, S. Fabrication of enclosed SU-8 tips for electrospray ionization-mass spectrometry. Electrophoresis 2005, 26, 4691-4702. [CrossRef]

19. Sikanen, T.; Tuomikoski, S.; Ketola, R.A.; Kostiainen, R.; Franssila, S.; Kotiaho, T. Fully microfabricated and integrated SU-8-based capillary electrophoresis-electrospray ionization microchips for mass spectrometry. Anal. Chem. 2007, 79, 9135-9144. [CrossRef]

20. Frederico, S.; Hibert, C.; Fritschi, R.; Fluckiger, P.; Renaud, P.; Ionescu, A.M. Silicon sacrificial layer dry etching (SSLDE) for free-standing RF MEMS architectures. In Proceedings of the Sixteenth Annual International Conference on Micro Electro Mechanical Systems, Kyoto, Japan, 23-23 January 2003; pp. 570-573. [CrossRef]

21. Chung, C.; Allen, M. Uncrosslinked SU-8 as a sacrificial material. J. Micromech. Microeng. 2005, 15, N1-N5. [CrossRef]

22. Patel, J.N.; Kaminska, B.; Gray, B.L.; Gates, B.D. PDMS as a sacrificial substrate for SU-8-based biomedical and microfluidic applications. J. Micromech. Microeng. 2008, 18, 095028. [CrossRef]

23. Luo, C.; Govindaraju, A.; Garra, J.; Schneider, T.; White, R.; Currie, J.; Paranjape, M. Releasing SU-8 structures using polystyrene as a sacrificial material. Sens. Actuators A Phys. 2004, 114, 123-128. [CrossRef]

24. Lau, K.H.; Giridhar, A.; Harikrishnan, S.; Satyanarayana, N.; Sinha, S.K. Releasing high aspect ratio SU-8 microstructures using AZ photoresist as a sacrificial layer on metallized Si substrates. Microsyst. Technol. 2013, 19, 1863-1871. [CrossRef]

25. Song, I.; Ajmera, P.K. Use of a photoresist sacrificial layer with SU-8 electroplating mould in MEMS fabrication. J. Micromech. Microeng. 2003, 13, 816-821. [CrossRef]

26. Haefliger, D.; Nordström, M.; Rasmussen, P.A.; Boisen, A. Dry release of all-polymer structures. Microelectron. Eng. 2005, 78-79, 88-92. [CrossRef]

27. Kim, B.J.; Liebau, M.; Huskens, J.; Reinhoudt, D.N.; Brugger, J. A self-assembled monolayer-assisted surface microfabrication and release technique. Microelectron. Eng. 2001, 57-58, 755-760. [CrossRef]

28. Schmidt, M.P.; Oseev, A.; Engel, C.; Brose, A.; Schmidt, B.; Hirsch, S. Flexible free-standing SU-8 microfluidic impedance spectroscopy sensor for 3-D molded interconnect devices application. J. Sens. Sens. Syst. 2016, 5, 55-61. [CrossRef]

29. Abgrall, P.; Lattes, C.; Conédéra, V.; Dollat, X.; Colin, S.; Gué, A.M. A novel fabrication method of flexible and monolithic 3D microfluidic structures using lamination of SU-8 films. J. Micromech. Microeng. 2006, 16, 113-121. [CrossRef]

30. Bohl, B.; Steger, R.; Zengerle, R.; Koltay, P. Multi-layer SU-8 lift-off technology for microfluidic devices. J. Micromech. Microeng. 2005, 15, 1125-1130. [CrossRef]

31. Sikanen, T.; Tuomikoski, S.; Ketola, R.; Kostiainen, R.; Sami Franssila, S.; Kotiaho, T. Analytical characterization of microfabricated SU-8 emitters for electrospray ionization mass spectrometry. J. Mass Spectrom. 2008, 43, 726-735. [CrossRef]

32. PMGI Datasheet; Microchem: Newton, MA, USA. Available online: http://microchem.com/pdf/PMGIResists-data-sheetV-rhcedit-102206.pdf (accessed on 21 November 2015).

33. OmniCoat Datasheet; Microchem: Newton, MA, USA. Available online: http://microchem.com/pdf/ OMNICOAT.pdf (accessed on 11 March 2016).

34. Technistrip Datasheet; Microchem: Newton, MA, USA. Available online: www.microchemicals.com/ products / remover_stripper/technistrip_ni555.html (accessed on 14 February 2017).

35. Barber, R.; Ghantasala, M.; Divan, R.; Mancini, D.; Harvey, E. Study of stress and adhesion strength in SU-8 resist layers on silicon substrate with different seed layers. J. Micro/Nanolithogr. MEMS MOEMS 2007, 6, 033006. [CrossRef] 
36. Young, S.; Weston, D.; Dauksher, B.; Mancini, D.; Pacheco, S.; Zurcher, P.; Miller, M. A novel low-temperature method to fabricate MEMS resonators using PMGI as a sacrificial layer. J. Micromech. Microeng. 2005, 15, 1824-1830. [CrossRef]

37. Valenc, M.; Atallah, T.; Castro, D.; Conchouso, D.; Un, K.A. Development of untethered SU-8 polymer scratch drive microrobots. In Proceedings of the 2011 IEEE 24th International Conference on Micro Electro Mechanical Systems, Cancun, Mexico, 23-27 January 2011; pp. 1221-1224.

38. Foulds, I.G.; Johnstone, R.W.; Parameswaran, M. Polydimethylglutarimide (PMGI) as a sacrificial material for SU-8 surface-micromachining. J. Micromech. Microeng. 2008, 18, 075011. [CrossRef]

39. Lipponen, K.; Tähkä, S.; Sikanen, T.; Jokinen, V.; Tatikonda, A.; Franssila, S.; Kostiainen, R.; Kotiaho, T. Thiol-ene micropillar array electrospray ionization platform for zeptomole level bioanalysis. Analyst 2017, 142, 2552-2557. [CrossRef] [PubMed]

(C) 2018 by the authors. Licensee MDPI, Basel, Switzerland. This article is an open access article distributed under the terms and conditions of the Creative Commons Attribution (CC BY) license (http://creativecommons.org/licenses/by/4.0/). 